\title{
Real-time physiologic monitoring and physician feedback: Are we ready?
}

\author{
Ekta Khemani, MD, MSc, FRCPC
}

Received: 5 October 2016/Revised: 12 October 2016/Accepted: 14 October 2016/Published online: 24 October 2016

(C) Canadian Anesthesiologists' Society 2016

In this edition of the Journal, Görges et al. present a quality improvement (QI) study on feedback to anesthesiologists regarding their management of intraoperative temperature monitoring. ${ }^{1}$ Spine anesthesiologists at their centre were provided with individual and group feedback on two variables for patients undergoing scoliosis surgery, namely, the percentage of time that their patients were hypothermic and the time delay from the start of the case to the first temperature monitoring (i.e., the primary outcome). While the authors did not find a difference in clinical outcomes, the delay to temperature monitoring in the operating room was reduced when anesthesiologists were given individual and group data on intraoperative temperature. Furthermore, the authors reasoned that the reduction in time to monitor intraoperative temperature offers some evidence that providing feedback data on intraoperative vital signs may reduce physician variability and could eventually contribute to improved perioperative outcomes. ${ }^{1}$

The concept of physician feedback as a QI tool is becoming increasingly popular. Some have argued that physician feedback should play an integral role in clinical practice for anesthesiologists, as it not only provides QI opportunities but also offers an opportunity for reflective and self-directed learning. ${ }^{2}$ A review by Kaye et al. even suggests the idea of a physician "report card" that could provide feedback to an anesthesiologist and perhaps play a central role in patient satisfaction and evidence-based healthcare. $^{2}$

E. Khemani, MD, MSc, FRCPC ( $)$

Department of Anesthesia and Perioperative Medicine,

Western University, London, ON, Canada

e-mail: Ekta.Khemani@1hsc.on.ca
In an era of balancing increasing healthcare costs while maintaining high-quality and patient-centred care, a focus on continuous QI is imperative. Quality improvement initiatives are a prominent area of interest to healthcare organizations, clinical practice, and research initiatives. Various QI endeavours have been shown to reduce operating room inefficiencies, improve antibiotic compliance, and reduce postoperative pneumonias. ${ }^{3}$ An understanding of the barriers to implementation is paramount to the success of these QI enterprises. Simply monitoring data without providing feedback, lack of trust in data, and lack of motivation have all been cited as reasons for ineffective QI buy-in and implementation. ${ }^{3}$

The study by Görges et al. addresses such common barriers by providing feedback through the use of statistical control processes. Nevertheless, unlike a traditional audit that examines snapshot data over a brief amount of time, the use of a continuous form of data created through run charts (which display observed data over a set time sequence - typically a year), creates a data-rich environment for assessing current practice over time and evaluating the impact of changes to the care system. ${ }^{3}$ Individualized feedback using such data can be a motivating factor for clinicians by providing objective data on clinical performance. ${ }^{3}$ Moreover, Görges et al. speculated that clinicians' intrinsic competitiveness through group feedback drove at least some of the improvements observed. ${ }^{1}$ It is possible that the provision of feedback results in a Hawthorne effect - i.e., that the knowledge of being measured actually changes behaviour. While this may affect the external validity, QI studies are largely observational in design with the goal to modify current practice at a single organization.

Physician feedback relies on the basic premise that data on physician performance will drive change. Implicit to 
this argument, however, is that the information is not used for punitive measures but rather for continuous QI and learning. Studies investigating why physicians do not report adverse events cite reasons such as fear of liability, loss of reputation, and peer disapproval as particularly strong motivators. ${ }^{4}$ Therefore, central to success of any QI program that incorporates physician feedback on performance is the change in culture that must accompany it. In the study by Görges et al., each anesthesiologist was given a unique identifier for QI purposes known only to them. ${ }^{1}$ The authors used this approach to maintain anonymity and perhaps cultivate a safe learning environment.

Individualized and group feedback requires us to embrace our own shortcomings and to accept our imperfections to drive improvement. As hospitals and departments of anesthesia continue to examine QI strategies to reduce costs and improve patient care, feedback without fear of punitive measures should be the catalyst for improvement. As clinicians, we must undergo a cultural shift from "shame and blame" to high-quality continuous improvement focused on patient-centred care. Are we ready?

\section{Le monitorage \\ physiologique en temps réel et les rétroactions au médecin: sommes-nous prêts?}

Dans ce numéro du Journal, Görges et coll. présentent une étude d'amélioration de la qualité fondée sur les rétroactions aux anesthésiologistes concernant leur prise en charge du monitorage peropératoire de la température. ${ }^{1}$ Dans un centre hospitalier, les anesthésiologistes spécialisés dans l'anesthésie du rachis ont reçu des rétroactions individuelles et de groupe au sujet de deux variables affectant les patients subissant une chirurgie de scoliose, soit le pourcentage de temps pendant lequel leurs patients étaient en hypothermie, et le laps de temps entre le début du cas et la première prise de température (le critère d'évaluation principal de l'étude). Bien que les auteurs n'aient pas observé de différence en matière de pronostics cliniques, ils ont toutefois noté une réduction du délai avant le monitorage de la température en salle d'opération après la réception, par les anesthésiologistes, des données individuelles et de groupe concernant la température peropératoire. En outre, selon les auteurs, la réduction de temps avant la prise de température peropératoire pourrait étayer l'hypothèse que des données rétroactives sur les signes vitaux peropératoires pourraient réduire la variabilité entre médecins et ainsi contribuer à de meilleurs pronostics périopératoires.

L'idée de la rétroaction au médecin en tant qu'outil d'amélioration de la qualité gagne chaque jour en popularité. Pour certains, la rétroaction au médecin devrait faire partie intégrante de la pratique clinique des anesthésiologistes : en effet, cet outil offre non seulement des occasions d'améliorer la qualité des prestations, mais il constitue également une occasion d'apprentissage autonome et réfléchi. ${ }^{2}$ Un compte rendu de Kaye et coll. propose même l'idée d'un 'bulletin scolaire' du médecin, qui présenterait des rétroactions à l'anesthésiologiste; selon ces auteurs, ce bulletin pourrait être le pivot de la satisfaction des patients et des soins de santé fondés sur des données probantes. ${ }^{2}$

Dans un âge où l'on tente d'équilibrer des coûts de santé toujours croissants et de maintenir des soins de santé de qualité élevée et centrés sur le patient, il est primordial de se concentrer sur une amélioration constante de la qualité. Les initiatives d'amélioration de la qualité sont un important domaine d'intérêt pour les organismes de soins de santé, la pratique clinique et les initiatives de recherche. Plusieurs initiatives d'amélioration de la qualité ont permis de réduire les inefficiences en salle d'opération, d'améliorer l'observance des antibiotiques, et de réduire les pneumonies postopératoires. ${ }^{3}$ Une bonne compréhension des obstacles à la mise en œuvre de telles tentatives d'amélioration de la qualité est absolument essentielle à leur réussite. La simple récolte de données sans rétroactions subséquentes, le manque de confiance dans les données, et le manque de motivation sont quelques-unes des raisons invoquées pour expliquer le manque de soutien de telles initiatives et une mise en œuvre inefficace. ${ }^{3}$

L'étude de Görges et coll. aborde ces obstacles communs en fournissant des rétroactions grâce au recours à des processus de contrôle statistiques. Toutefois, à la différence d'un contrôle de qualité conventionnel qui examine une sélection de données sur une brève période, l'utilisation de données en continu présentées via des graphiques de séquences (qui exposent les données observées sur une séquence temporelle déterminée - en général une année), crée un environnement riche en données dans lequel on peut évaluer la pratique actuelle au fil du temps et évaluer l'impact de changements apportés au système de soins. ${ }^{3}$ Les rétroactions personnalisées fondées sur de telles données peuvent motiver les cliniciens en leur présentant des données objectives sur leur performance clinique. ${ }^{3}$ De plus, Görges et coll. ont émis l'hypothèse que l'esprit de compétition intrinsèque des cliniciens, alimenté via les rétroactions de groupe, a probablement motivé certaines des améliorations 
observées. $^{1}$ Il est possible qu'en fournissant des rétroactions, on entraîne un effet Hawthorne - c'est-à-dire que les résultats ne sont pas dus aux facteurs expérimentaux, mais au fait que les sujets ont conscience de participer à une expérience dans laquelle ils sont testés, ce qui modifie leur comportement. Bien que cet effet puisse affecter la validité externe de l'expérience, les études d'amélioration de la qualité sont, la plupart du temps, conçues sur un modèle observationnel, et leur but est de modifier la pratique actuelle dans une institution donnée.

Les rétroactions aux médecins s'appuient sur la prémisse de base que les données sur la performance des médecins constitueront un moteur de changement. Il est toutefois implicitement entendu que ces informations ne seront pas utilisées à des fins punitives, mais plutôt dans un objectif d'amélioration constante de la qualité et d'apprentissage. Les études s'intéressant aux raisons pour lesquelles les médecins ne rapportent pas les événements indésirables citent la crainte d'être tenus responsables, la perte de réputation et la désapprobation des pairs comme certaines des raisons qui motivent leur silence. ${ }^{4}$ C'est pourquoi l'un des éléments clés de la réussite de tout programme d'amélioration de la qualité intégrant des rétroactions aux médecins sur leur performance est le changement de culture qui doit accompagner un tel programme. Dans l'étude de Görges et coll., chaque anesthésiologiste a reçu un identifiant unique aux fins d'amélioration de la qualité. Cet identifiant n'était connu que de l'anesthésiologiste et permettait de maintenir l'anonymat tout en cultivant un environnement d'apprentissage sécuritaire.

Les rétroactions personnalisées et de groupe requièrent de nous que nous admettions nos défauts et acceptions nos imperfections afin de faire place aux améliorations. Alors que les hôpitaux et les départements d'anesthésie continuent d'explorer diverses stratégies d'amélioration de la qualité pour réduire les coûts et améliorer les soins aux patients, les rétroactions, sans crainte de mesures punitives, devraient être un catalyseur d'améliorations. En tant que cliniciens, nous devons modifier notre paradigme culturel de «honte et blâme » pour qu'il devienne un paradigme d'améliorations constantes et de qualité élevée concentrées sur des soins centrés sur les patients. Sommes-nous prêts?

Conflicts of interest None declared.

Editorial responsibility This submission was handled by Dr. Hilary P. Grocott, Editor-in-Chief, Canadian Journal of Anesthesia.

Conflit d'intérêt Aucun.

Responsabilité éditoriale Cet article a été traité par Dr Hilary P. Grocott, rédacteur en chef, Journal canadien d'anesthésie.

\section{References}

1. Görges $G$, West $N C$, Whyte $S D$. Using physiological monitoring data for performance feedback: an initiative using thermoregulation metrics. Can J Anesth 2017; 64. DOI: 10.1007/s12630-016-0762-3.

2. Kaye $A D$, Okanlawon OJ, Urman RD. Clinical performance feedback and quality improvement opportunities for perioperative physicians. Adv Med Educ Pract 2014; 5: 115-23.

3. Benn J, Arnold $G$, Wei I, Riley $C$, Aleva $F$. Using quality indicators in anesthesia: feeding back data to improve care. $\mathrm{Br} \mathrm{J}$ Anaesth 2012; 109: 80-91.

4. Leape LL. Reporting of adverse events. N Engl J Med 2002; 347 : 1633-8. 